\title{
Dynamic Mechanical Properties of Tissue after Long-Term Implantation of Collagen and Polypropylene Meshes in Animal Models
}

\author{
Michail G. Christodoulou ${ }^{1}$, Apostolos Papalois², Dionysios Mouzakis ${ }^{3}$, Stefanos Zaoutsos ${ }^{3}$, \\ Theodoros Kouranos $^{1}$, Miltiadis Seferlis ${ }^{1}$, Charilaos Katsifotis ${ }^{1}$, Angelos Liapis ${ }^{4}$ \\ ${ }^{1}$ Department of Urology, General Hospital of Athens Polycliniki, Athens, Greece \\ ${ }^{2}$ Experimental Research Center ELPEN, Athens, Greece \\ ${ }^{3}$ Department of Mechanical Engineering, Technological Educational Institute of Larissa, Larissa, Greece \\ ${ }^{4}$ 2nd Department of Obstetrics and Gynecology, Aretaieio Hospital, University of Athens, Athens, Greece \\ Email: michailchristodoulou79@gmail.com, apapalois@elpen.gr,mouzakis@teilar.gr, szaoutsos@teilar.gr, \\ koura1302@yahoo.gr,milsef@hotmail.com, ch.katsifotis@gmail.com, urodyn@aretaieio.uoa.gr
}

Received May 20, 2013; revised June 18, 2013; accepted June 25, 2013

Copyright (C) 2013 Michail. G. Christodoulou et al. This is an open access article distributed under the Creative Commons Attribution License, which permits unrestricted use, distribution, and reproduction in any medium, provided the original work is properly cited.

\begin{abstract}
Purpose: Pelvic floor reconstructive surgery has grown significantly in recent years. A wide variety of available types of meshes exist but the safety and success has not been adequately proven. We sought to evaluate the effects on dynamic biomechanical properties of tissue after long-term implantation of synthetic and biological grafts. Methods: A total of 96 New Zealand white female rabbits (approximately $3 \mathrm{~kg}$ ) were used, 72 of which were surgically implanted with acellular, collagen mesh $(n=36)$ or nonabsorbable monofilament polypropylene mesh $(n=36)$. There was a no mesh-rupture of fascia group $(\mathrm{n}=12)$ and a second, no-mesh, no-fascia rupture control group $(\mathrm{n}=12)$. In the 59 rabbits, of 72 (13 died) tissue was harvested 3 months $(n=24), 6$ months $(n=23)$ and 9 months $(n=12)$ later, while in the fascia rupture group, tissue was harvested 6 months later. Tissue samples $(2 \times 2 \mathrm{~cm})$ underwent dynamic mechanical analysis (DMA) testing during which the dynamic rigidity and tissue damping capacities were measured. The statistical analysis was performed with General Linear Model with Tukeys post hoc testing (sPss v.17.0). Results: With respect to mesh type, the rabbit tissue in which polypropylene mesh was used showed the greatest dynamic rigidity. Those with biological mesh delivered the lowest rigidity results, while the two other groups had almost similar behavior. The meshes exhibited their highest relative dynamic tissue stiffening effect at 9 months. Conclusions: Biological mesh causes lower tissue rigidity, resulting in inferior mechanical response and thus seems to be inferior to polypropylene.
\end{abstract}

Keywords: Biomechanical Properties; Tissue Stiffening; Collagen-Polypropylene Meshes

\section{Introduction}

Disorders of the pelvic floor encompass a wide spectrum of interrelated clinical entities, including pelvic organ prolapse and incontinence. In addition to the physical symptoms that accompany these disorders, there is an important emotional effect, which includes social isolation, anxiety and depression [1]. Pelvic organ prolapse affects almost one third of premenopausal and approximately one half of postmenopausal women [2,3]. Every year, approximately 135,000 women undergo surgery for urinary incontinence [4] and 225,000 for pelvic organ prolapse at a cost of greater than $\$ 1$ billion per year in the United States [4,5].

Mechanical failure of the tissues in these anatomical areas is one of the causes [6] as the muscles and ligaments of the pelvis cannot provide adequate support, for which sufficient connective tissue is paramount [7]. On the other hand, reconstructive procedures for the pelvic floor have increased exponentially in the past 20 years and the recent literature has shown increasing interest in the use of biological and synthetic mesh $[8,9]$. The principle behind using such grafts in reconstructive surgery is reinforcement of existing tissues with materials that are safe, biologically compatible and provide suitable anatomical and functional results. An ideal mesh must be inert, non-carcinogenic, resistant to tension, sterilizable, non-allergenic and non-inflammatory, unaffected by the body's tissues and of course affordable [10]. In the effort 
to find an ideal mesh, biomechanics, i.e. the study of mechanical properties of tissue and ligaments, could allow us a better understanding of the normal function of the tissues and of the effect of the treatment being applied.

The goal of this study was the appraisal of the biomechanical properties of rabbit tissues after the implantation of biological and synthetic mesh and their long-term stay in the abdominal rectus muscle.

\section{Material and Methods}

Permission for this study was obtained from the Veterinary Service of Eastern Attica Prefecture and from the Institutional Review Board of Aretaion Hospital (Reference number: N-52/20-12-2005). A total of 96 white, female New Zealand rabbits weighing $3-3.5 \mathrm{~kg}$ were used. All established rules regarding the humane handling of animals and animal products were followed and they were physically sheltered at the Experimental Research Center of ELPEN. The rabbits, in which mesh was placed, were divided into two groups. In the first group $(\mathrm{n}=36)$ porous collagen mesh (Pelvisoft Acellular Collagen Biomesh C. R. Bard Inc, code number 481812) was placed into the rectus muscle, while in the second group $(n=36)$ it was a monofilament polypropylene mesh (Avaulta Solo Synthetic mesh C. R. Bard Inc, code number 486100). The meshes measuring $2 \times 2 \mathrm{~cm}$ were sutured tensionfree with four 5 - 0 Prolene stitches. Subsequently, each group was divided into subsets of 12 each and sacrificed at 3,6 and 9 months (post-operative) respectively. There were also two other groups; in one $(n=12)$ there was only surgical division of the fascia of the rectus abdominis muscle without any mesh placement (fascia rupture), while in the other $(\mathrm{n}=12)$ neither mesh was placed nor surgical division of the fascia was done (control group). These groups (fascia rupture and control group) were sacrificed at 6 months and 0 months respectively (Table 1).

Subsequently, a $2 \times 2 \mathrm{~cm}$ sample of tissue in the region of the mesh placement (without the mesh) or fasciotomy was removed and then preserved at $-80^{\circ} \mathrm{C}$ until the biomechanical study with the Dynamic Mechanical Analyzer, DMA Q800 (by TA Instruments). The tissues were isothermally maintained at $37^{\circ} \mathrm{C}$ and were subjected to sine-wave dynamic tension under a spectrum of low

Table 1. Time period and number of rabbits being sacrificed from the $\mathbf{4}$ different groups.

\begin{tabular}{|c|c|c|c|c|}
\hline $\begin{array}{l}\text { Four groups/ } \\
\text { sacrifice time (months) }\end{array}$ & 0 month & 3 months & 6 months & 9 months \\
\hline 1. Collagen mesh & & 12 rabbits & 12 rabbits & 6 rabbits \\
\hline 2. polypropylene mesh & & 12 rabbits & 11 rabbits & 6 rabbits \\
\hline 3. Fascia rupture & & & 12 rabbits & \\
\hline 4. No mesh-no rupture & 12 rabbits & & & \\
\hline
\end{tabular}

frequencies $(0.1-15 \mathrm{~Hz})$. DMA testing allows materials to be tested within ranges of frequencies which correspond to the actual loading conditions during their operation. Therefore frequencies of $0.1-15 \mathrm{~Hz}$ were used in order to simulate actual loading of tissue under static, e.g. standing (low frequencies in the area of $0.1-1 \mathrm{~Hz}$ ) or dynamic conditions like running, jumping etc (frequencies $>1-15 \mathrm{~Hz}$ ). Tissue was loaded under such strain and stress levels so as to correspond to linear elastic viscoelastic regime. The static force (preload) was $0.3 \mathrm{New}-$ ton and the oscillation amplitude 80 micrometers $(=0.08$ $\mathrm{mm})$. The main three viscoelastic parameters were recorded, i.e. the capacity of the material to store elastic energy (storage modulus) or else its dynamic stiffness, the loss of elastic energy per load cycle (loss modulus) and the ability of the material to absorb oscillation and impact $(\tan \delta)$. For each group three different measurements were made by dividing each specimen into three equal parts (Number of cycles: 4 until data stabilization set by the DMA test set-up). The rationale behind employing DMA is the great sensitivity of the method to detect changes and the ability of the instrument to impose dynamic loading on the tissue specimens at various strains and frequencies.

The statistical analysis was performed with General Linear Model with Tukeys post hoc testing (sPss v.17.0). Differences were considered statistically significant when they reached a $\mathrm{P}$ value of $<0.05(\mathrm{P}<0.05)$.

\section{Results}

During the study, 10 rabbits showed mild subcutaneous inflammation ( 3 with biological mesh, 7 with synthetic), which was successfully treated with antibiotics, whereas 13 rabbits died and were excluded from analysis. In those 13 rabbits biological mesh had been placed in 6 of them and they belonged to the group to be sacrificed at 9 months. Polypropylene mesh had been placed in the remaining 7, six of which were to be sacrificed at 9 months, and one at 6 months. As for the 13 excluded participants, a possible connection to the mesh arose in only two (a subcutaneous tumor and an abscess, with biological and synthetic mesh respectively) on autopsy.

Considering the independent variables of the viscoelastic parameters (type of mesh, time, frequency), it became evident that primarily the type of mesh, and also time, affected the biomechanical properties of the tissues to a statistically significant degree (Table 2).

The tissue from rabbits that received polypropylene mesh showed the greatest dynamic stiffness (storage moduli). This implies that this tissue is resisting straining at a higher level than other samples, or in plain words that the corresponding specimens exhibited the highest rigidity. The collagen mesh showed the least rigidity among the four groups (synthetic mesh, biological mesh, fascia 
Table 2. Percentage of total variation explained by correlation factor $(\%)$.

\begin{tabular}{lccc}
\hline & Storage modulus & Loss modulus & Tan $\delta$ \\
\hline Mesh type & $4.04 \mathrm{~b}$ & $5.09 \mathrm{~b}$ & $35.13 \mathrm{~b}$ \\
Time & $0.35 \mathrm{a}$ & $0.32 \mathrm{a}$ & $0.98 \mathrm{~b}$ \\
Frequency & $1.46 \mathrm{~b}$ & $1.30 \mathrm{~b}$ & - \\
Mesh * Time & $1.00 \mathrm{~b}$ & $1.11 \mathrm{~b}$ & $3.55 \mathrm{a}$ \\
Mesh * Freq. & - & - & $52.53 \mathrm{~b}$ \\
\hline
\end{tabular}

(-) Not statistically significant impact. Levels: a: 0.05 Significance, b: 0.001 Significance. Storage modulus: The capacity of the material to store elastic energy. Loss modulus: The loss of elastic energy per load cycle. Tan $\delta$ : The ability of the material to absorb oscillation and impact.

rupture, control group), while the 2 groups, in which no mesh was placed, showed no significant difference between them (Table 3 and Figure 1). The greatest stiffening of tissue, irrespectively of mesh type, was achieved at 9 months (Table 3 and Figure 2). At 3 and 6 months, the increase in dynamic stiffness for the three groups (biological mesh, synthetic mesh, fasciotomy) was not statistically significant.

\section{Discussion}

It is known that pelvic floor reconstructive surgery has grown significantly in recent years and several studies have been performed trying to determine which mesh is most suitable for implantation. These have been in animal models and focused on the dynamic properties of the mesh itself and not the tissue. In this study, the biomechanical properties of the tissue from rabbits were assessed, after the use of biological and synthetic mesh, and it appears that synthetic mesh is superior, as it provokes tissue stiffening to a greater degree. This is supported in the current literature with randomized controlled studies [10], which have stood the test of time.

Deprest et al. [11] found that collagen mesh causes less inflammatory reaction, delayed deposition of collagen and slower strengthening of tension than synthetic mesh. They believed this difference was due to polypropylene mesh being porous, whereas collagen mesh is not. In a more recent study of theirs [12] they placed such pores preoperatively in the biological mesh and observed more rapid neovascularization, deposition of collagen and strengthening of tissue. In our study, porous biological mesh was used, which proved inferior to the synthetic one in reinforcing the tissue of the animal model, based on our results.

In an effort to find a mesh that would be associated with fewer complications, such as erosion, infection and pain, as compared to synthetic ones, several clinical studies have been carried out regarding pelvic floor surgery. Altman et al. [13] reported that there were no complications when using biological mesh, Quiroz et al. [14] reported a complication rate of about $24 \%$ with collagen
Table 3. Estimated marginal means for DMA parameters with respect to mesh type and indwell time.

\begin{tabular}{ccccccc}
\hline & $\begin{array}{c}\text { Sample } \\
\text { type }\end{array}$ & Meana & $\begin{array}{c}\text { Std. } \\
\text { Error }\end{array}$ & $\begin{array}{c}\text { Indw. Time } \\
\text { [months] }\end{array}$ & Meana & $\begin{array}{c}\text { Std. } \\
\text { Error }\end{array}$ \\
\hline Storage & 1 & 0.134 & 0.048 & 0 & 0.373 & 0.071 \\
$\begin{array}{c}\text { Modulus } \\
{[\mathrm{MPa}]}\end{array}$ & 2 & 0.933 & 0.049 & 3 & 0.520 & 0.051 \\
& 4 & 0.551 & 0.073 & 6 & 0.441 & 0.042 \\
& 1 & 0.373 & 0.071 & 9 & 0.695 & 0.073 \\
Loss & 2 & 0.025 & 0.010 & 0 & 0.076 & 0.014 \\
Modulus & 3 & 0.109 & 0.015 & 6 & 0.097 & 0.008 \\
{$[\mathrm{MPa}]$} & 4 & 0.076 & 0.014 & 9 & 0.151 & 0.015 \\
& 1 & 0.141 & 0.004 & 0 & 0.154 & 0.006 \\
& 2 & 0.410 & 0.004 & 3 & 0.246 & 0.004 \\
Tan $\delta$ & 3 & 0.262 & 0.006 & 6 & 0.280 & 0.004 \\
& 4 & 0.154 & 0.006 & 9 & 0.291 & 0.006 \\
\hline
\end{tabular}

Sample type: 1. Collagen mesh; 2. Polypropylene mesh; 3 . Fascia rupture; 4. No mesh-no rupture. MPa: megapascal $\left[=1 \mathrm{~N} / \mathrm{mm}^{2}\right]$.

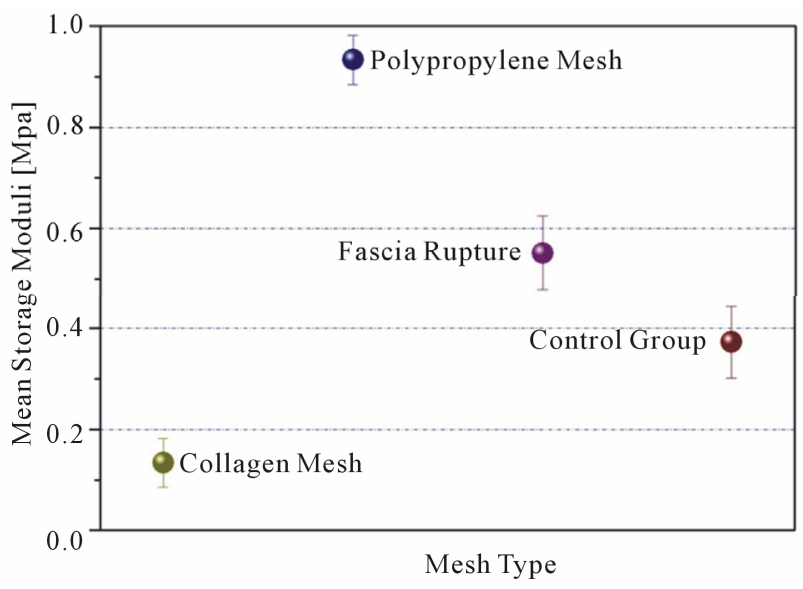

Figure 1. Dynamic stiffness related to 4 groups.

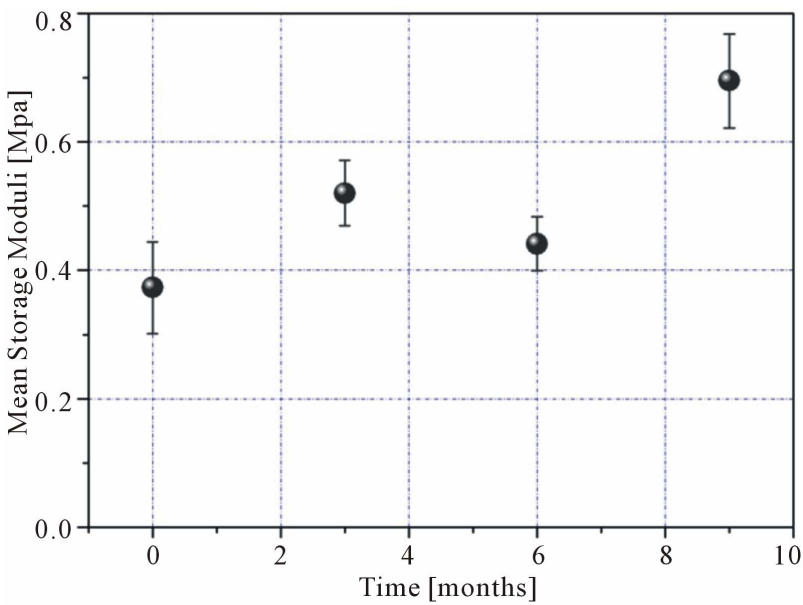

Figure 2. Dynamic stiffness related to time (irrespectively of mesh type). 
mesh, while Deprest et al. [15] observed that biological mesh not only does not reduce complications compared to synthetic, but also has high, almost unacceptable rates of failure. In our study, mild subcutaneous inflammation was observed in 10 rabbits, which was treated successfully with antibiotics, while in the 13 subjects that died and were excluded from the study, there was a possible association with the mesh noted only in two (a subcutaneous tumor and an abscess) on autopsy.

In our study, the collagen mesh was inferior in tissue reinforcement, not only compared to the synthetic mesh, but also compared to the two groups in which no mesh was placed. This paradoxical finding may be explained by the fact that collagen mesh has been proven to undergo, to varying degrees, premature decomposition, for reasons unclear to date $[10,16-18]$, and this might lead to a reduction in its biomechanical properties. Another explanation may be that the higher concentration of collagen in the area might decrease the micromechanical properties of the surrounding tissue.

There are limitations in our study as well. The biomechanical properties of the tissues were observed under dynamic loading, which does not occur to such a degree (frequencies up to $15 \mathrm{~Hz}$ ) under normal conditions and therefore in future studies, the range of physiological tension of the vaginal wall must be considered [16,19-21]. In addition, rabbits exhibit increased collagenolytic activity and thereby perhaps cause more rapid decomposition of collagen mesh as compared to humans [16,17], although there is a study in the literature reporting similar decomposition of collagen mesh in women [18].

In conclusion, despite a wide variety of available types of mesh, the safety and success of any one of these has not been adequately proven. Further studies are needed to evaluate these parameters in order to show which mesh is ideal. We believe that our study contributes to better evaluating the biomechanical properties of mesh and that this knowledge may assist in the improved design of future studies.

\section{Acknowledgements}

The authors would like to acknowledge the unrestricted grant of the mesh from the company C. R. Bard Inc.

\section{REFERENCES}

[1] S. D. Abramowitch, A. Feola, Z. Jallah and Pa. Moalli, "Tissue Mechanics, Animal Models, and Pelvic Organ Prolapse: A Review," European Journal of Obstetrics \& Gynecology and Reproductive Biology, Vol. 144, No. S1, 2009, pp. S146-S158. doi:10.1016/j.ejogrb.2009.02.022

[2] A. L. Olsen, V. J. Smith, J. O. Bergstrom, J. C. Colling and A. L. Clark, "Epidemiology of Surgically Managed Pelvic Organ Prolapse and Urinary Incontinence," $\mathrm{Ob}$ - stetrics \& Gynecology, Vol. 89, No. 4, 1997, pp. 501-506. doi:10.1016/S0029-7844(97)00058-6

[3] G. R. Rogers, A. Villarreal, D. Kammerer-Doak and C. Qualls, "Sexual Function in Women with and without Urinary Incontinence and/or Pelvic Organ Prolapse," International Urogynecology Journal and Pelvic Floor Dysfunction, Vol. 12, No. 6, 2001, pp. 361-365. doi:10.1007/s001920170012

[4] L. L. Subak, L. E. Waetjen, S. van den Eeden, D. H. Thom, E. Vittinghoff and J. S. Brown, "Cost of Pelvic Organ Prolapse Surgery in the United States," Obstetrics \& Gynecology, Vol. 98, No. 4, 2001, pp. 646-651. doi:10.1016/S0029-7844(01)01472-7

[5] J. S. Brown, L. E. Waetjen, L. L. Subak, D. H. Thom, S. Van den Eeden and E. Vittinghoff, "Pelvic organ Prolapse Surgery in the United States, 1997," American Journal of Obstetrics \& Gynecology, Vol. 186, No. 4, 2002, pp. 712716. doi:10.1067/mob.2002.121897

[6] B. Chen, Y. Wen and M. L. Polan, "Elastolytic Activity in Women with Stress Urinary Incontinence and Pelvic Organ Prolapse," Neurourology and Urodynamics, Vol. 23, No. 2, 2004, pp. 119-126. doi:10.1002/nau.20012

[7] A. Liapis, P. Bakas, A. Pafiti, D. Hassiakos, M. FrangosPlemenos and G. Creatsas, "Changes in the Quantity of Collagen Type I in Women with Genuine Stress Incontinence," Urological Research, Vol. 28, No. 5, 2000, pp. 323-326. doi: $10.1007 / \mathrm{s} 002400000120$

[8] C. Birch and M. M. Fynes, "The Role of Synthetic and Biological Prostheses in Reconstructive Pelvic Floor Surgery," Current Opinion in Obstetrics and Gynecology, Vol. 14, No. 5, 2002, pp. 527-535. doi:10.1097/00001703-200210000-00015

[9] C. Birch, "The Use of Prosthetics in Pelvic Reconstructive Surgery," Best Practice \& Research Clinical Obstetrics \& Gynaecology, Vol. 19, No. 6, 2005, pp. 979-991. doi:10.1016/j.bpobgyn.2005.08.013

[10] S. M. Jakus, A. Shapiro and C. D. Hall, "Biologic and Synthetic Graft Use in Pelvic Surgery: A Review," $\mathrm{Ob}$ stetrical \& Gynecological Survey, Vol. 63, No. 4, 2008, pp. 253-266. doi:10.1097/OGX.0b013e318166fb44

[11] F. Zheng, Y. Lin, E. Verbeken, F. Claerhout, M. Fastrez and J. Deprest, "Host Response after Reconstruction of Abdominal Wall Defects with Porcine Dermal Collagen in a Rat Model," American Journal of Obstetrics \& Gynecology, Vol. 191, No. 6, 2004, pp. 1961-1970. doi:10.1016/j.ajog.2004.01.091

[12] F. Zheng, E. Verbeken, D. de Ridder and J. Deprest, "Improved Surgical Outcome by Modification of Porcine Dermal Collagen Implant in Abdominal Wall Reconstruction in Rats," Neurourology and Urodynamics, Vol. 24, No. 4, 2005, pp. 362-368. doi:10.1002/nau.20148

[13] D. Altman, B. Anzen, S. Brismar, A. Lopez and J. Zetterström, "Long-Term Outcome of Abdominal Sacrocolpopexy Using Xenograft Compared with Synthetic Mesh," Urology, Vol. 67, No. 4, 2006, pp. 719-724. doi:10.1016/j.urology.2005.10.034

[14] L. H. Quiroz, R. E. Gutman, S. Shippey, G. W. Cundiff, T. Sanses and V. L. Handa, "Abdominal Sacrocolpopexy: 
Anatomic Outcomes and Complications with Pelvicol, Autologous and Synthetic Graft Materials," American Journal of Obstetrics \& Gynecology, Vol. 198, No. 5, 2008, pp. 557.e1-e5.

[15] F. Claerhout, D. De Ridder, D. Van Beckevoort, G. Coremans, J. Veldman and J. Deprest, "Sacrocolpopexy Using Xenogenic Acellular Collagen in Patients at Increased Risk for Graft-Related Complications," Neurourology and Urodynamics, Vol. 29, No. 4, 2010, pp. 563-567.

[16] L. M. Pierce, M. A. Grunlan, Y. Hou, S. S. Baumann, T. J. Kuehl and T. W. Muir, "Biomechanical Properties of Synthetic and Biologic Graft Materials Following LongTerm Implantation in the Rabbit Abdomen and Vagina," American Journal of Obstetrics \& Gynecology, Vol. 200, No. 5, 2009, pp. 549.e1-e8.

[17] F. Claerhout, G. Verbist, E. Verbeken, M. Konstantinovic, D. De Ridder and J. Deprest, "Fate of Collagen-Based Implants Used in Pelvic Floor Surgery: A 2-Year Follow-Up Study in a Rabbit Model," American Journal of Obstetrics \& Gynecology, Vol. 198, No. 1, 2008, pp. 94.e1-e6.

[18] S. Gandhi, L. M. Kubba, Y. Abramov, S. M. Botros, R. P.
Goldberg and P. K. Sand, "Histopathologic Changes of Porcine Dermis Xenografts for Transvaginal Suburethral Slings," American Journal of Obstetrics \& Gynecology, Vol. 192, No. 5, 2005, pp. 1643-1648. doi:10.1016/j.ajog.2004.11.044

[19] J. T. Goh, "Biomechanical and Biochemical Assessments for Pelvic Organ Prolapse," Current Opinion in Obstetrics and Gynecology, Vol. 15, No. 5, 2003, pp. 391-394. doi:10.1097/00001703-200310000-00007

[20] L. Lei, Y. Song and R. Chen, "Biomechanical Properties of Prolapsed Vaginal Tissue in Pre- and Postmenopausal Women," International Urogynecology Journal and Pelvic Floor Dysfunction, Vol. 18, No. 6, 2007, pp. 603-607. doi:10.1007/s00192-006-0214-7

[21] M. Cosson, E. Lambaudie, M. Boukerrou, P. Lobry, G. Crépin and A. Ego, "A Biomechanical Study of the Strength of Vaginal Tissues. Results on 16 Post-Menopausal Patients Presenting with Genital Prolapse," European Journal of Obstetrics \& Gynecology and Reproductive Biology, Vol. 112, No. 2, 2004, pp. 201-205. doi:10.1016/S0301-2115(03)00333-6 